\title{
Efeito Cardiodepressor do Acetato de Eugenil em Coração de Roedor
}

\author{
Cardiodepressive Effect of Eugenyl Acetate in Rodent Heart
}

\author{
Leisiane Pereira Marques, ${ }^{1}$ Samuel Santos Beserra, ${ }^{2}$ Danilo Roman-Campos, ${ }^{2}$ Antonio Nei Santana Gondim ${ }^{1,2}$ \\ Universidade do Estado da Bahia - Departamento de Educação, ${ }^{1}$ Salvador, BA - Brasil \\ Universidade Federal de São Paulo - Biofísica, ${ }^{2}$ São Paulo, SP - Brasil
}

\section{Resumo}

No presente trabalho investigou-se o efeito inotrópico do acetato de eugenil (AE), bem como sua ação sobre a corrente de $\mathrm{Ca}^{2+}$ do tipo $\mathrm{L}\left(\mathrm{I}_{\mathrm{Ca}, \mathrm{L}}\right)$. Os experimentos de contratilidade foram realizados em átrio esquerdo isolado de cobaia exposto às concentrações crescentes da droga (1 a $5.000 \mu \mathrm{M})$. O AE reduziu a força de contração atrial $\left(\mathrm{IC}_{50}=558 \pm 24,06 \mu \mathrm{M}\right)$ de modo dependente de concentração. O efeito do AE sobre a $\mathrm{I}_{\mathrm{Ca}, \mathrm{L}}$ também foi avaliado em cardiomiócitos ventriculares isolados de camundongos, utilizando-se a técnica de "patch-clamp". O AE apresentou um efeito inibitório $\left(\mathrm{IC}_{50}=1.337 \pm 221 \mu \mathrm{M}\right)$ sobre os canais de $\mathrm{Ca}^{2+}$ sensíveis à voltagem $\left(\mathrm{Ca}_{\mathrm{v}} 1.2\right)$. Em conclusão, o AE apesenta efeito cardiodepressor que se deve, pelo menos em parte, à diminuição da entrada de $\mathrm{Ca}^{2+}$ nos cardiomiócitos.

\section{Introdução}

As doenças cardiovasculares são um problema de saúde pública e estão entre as principais causas de óbitos no mundo. ${ }^{1}$ Nessa perspectiva, é crescente o interesse pela busca de novas substâncias com propriedades farmacológicas sobre o sistema cardiovascular, principalmente as de origem natural.

A planta Syzygium aromaticum (L.) Merr. \& L.M.Perry, popularmente conhecida como cravo-da-índia, é constituída por diversos compostos químicos que apresentam uma ampla gama de efeitos farmacológicos. O eugenol (Figura 1A) é o mais abundante composto bioativo encontrado no óleo essencial do cravo-da-índia, seguido pelo acetato de eugenil (AE) (Figura 1B). ${ }^{2}$

Estudos demonstraram que o eugenol apresenta atividade cardiodepressora em ratos ${ }^{3}$ e cobaias ${ }^{4}$ provavelmente devido à inibição da corrente de $\mathrm{Ca}^{2+}$ do tipo $\mathrm{L}\left(\mathrm{I}_{\mathrm{Ca}, \mathrm{L}}\right)$. Além disso, o eugenol atua como cardioprotetor. ${ }^{5}$

Apesar de diversos estudos abordarem as propriedades farmacológicas do eugenol sobre o coração, até o momento

\section{Palavras-chave}

Acetato de Eugenil; Contração Miocárdica; Syzygium Aromaticum, Ratos.

Correspondência: Antonio Nei Santana Gondim •

Universidade do Estado da Bahia - Departamento de Educação

Av. Universitária Vanessa Cardoso e Cardoso, s/n. CEP 46430-000

Guanambi, BA - Brasil

E-mail: agondim@uneb.br, agondim@unebb.br

Artigo recebido em 21/11/2019, revisado em 18/02/2020,

aceito em 16/03/2020

DOI: https://doi.org/10.36660/abc.20190823 não existem informações sobre a ação do AE no miocárdio. Dessa forma, o presente estudo descreve, pela primeira vez, os efeitos do AE sobre a contratilidade cardíaca e sua ação inibitória sobre a $\mathrm{I}_{\mathrm{Ca}, \mathrm{L}}$.

\section{Métodos}

\section{Animais}

Para os experimentos de contratilidade foram utilizadas cobaias machos e fêmeas (Cavia porcellus, 400-600g). Para os estudos eletrofisiológicos foram utilizados camundongos machos adultos $\mathrm{C} 57 \mathrm{Bl} / 6 \mathrm{~J}$. Todos os procedimentos foram aprovados pela Comissão de Ética para Uso de Animais (CEUA) da Universidade do Estado da Bahia (licença: 03/2017).

\section{Protocolos Experimentais}

\section{Avaliação do Efeito Inotrópico do AE}

O átrio esquerdo foi mantido em solução de Tyrode modificada $\left(10 \mathrm{~mL}, 36,5 \pm 0,5^{\circ} \mathrm{C}\right)$ com a seguinte composição (em mM): $140 \mathrm{NaCl} ; 5,4 \mathrm{KCl} ; 0,5 \mathrm{MgCl}_{2} ; 0,33 \mathrm{NaH}_{2} \mathrm{PO}_{4} ; 11$ glicose; 5 HEPES e 1,8 $\mathrm{CaCl}_{2}(\mathrm{pH}=7,4)$, aerado com oxigênio $(99,9 \%)$, estirado para uma tensão de $1 \mathrm{gF}$ e estimulado eletricamente ( $2 \mathrm{~Hz}, 100 \mathrm{~V}, 15 \mathrm{~ms})$. A força contrátil foi captada por um transdutor isométrico, sendo os sinais digitalizados $(512 \mathrm{~Hz})$ e armazenados em um computador. Os átrios esquerdos foram submetidos às concentrações crescentes de $\mathrm{AE}(1-5.000 \mu \mathrm{M}, 3$ a 5 minutos).

Para fazer a solução-estoque de AE (obtido da SigmaAldrich) foi usado dimetil sulfóxido (DMSO).

\section{Avaliação do Efeito do AE Sobre a Corrente de Cálcio Tipo L}

Cardiomiócitos ventriculares de camundongos C57Bl/6] foram enzimaticamente isolados. ${ }^{6}$ Para medir a corrente de $\mathrm{Ca}^{2+}$ do tipo $\mathrm{L}\left(\mathrm{I}_{\mathrm{Ca}, \mathrm{L}}\right)$ foi utilizada a técnica de patch-clamp $p^{7,8}$ no modo whole-cell voltage-clamp. A composição da solução interna (em mM) foi: $120 \mathrm{CsCl}, 10$ HEPES, 5 EGTA, 20 TEA$\mathrm{Cl}$ e $5 \mathrm{NaCl}(\mathrm{pH}=7,2 ; \mathrm{CsOH})$. O Tyrode foi usado como solução externa. As células foram mantidas em um potencial de membrara de $-80 \mathrm{mV}$, e depois foram submetidas a um pré-pulso que despolarizou a membrana para $-40 \mathrm{mV}$ (50ms). Em seguida a membrana foi despolarizada para $0 \mathrm{mV}(300 \mathrm{~ms}$, $0.1 \mathrm{~Hz}$ ). A amplitude da $\mathrm{I}_{\mathrm{Ca}, \mathrm{L}}$ foi medida pela diferença entre $\mathrm{O}$ final do pulso teste $(0 \mathrm{mV})$ e o pico. As células foram expostas ao $\mathrm{AE}$ (10-3.000 $\mu \mathrm{M}, 2-3$ minutos). Os sinais foram digitalizados $(5 \mathrm{kHz})$ e armazenados em computador. 


\section{Análise Estatística}

Os resultados são expressos com média \pm erro padrão da média e foram analisados estatisticamente empregando-se o teste " $\mathrm{t}$ " pareado na sua forma bicaudal (nível de significância: $\mathrm{p}<0,05)$.

\section{Resultados}

\section{Efeito do AE Sobre a Força de Contração Miocárdica}

Os traçados da Figura 1C mostram que o AE (700 $\mu \mathrm{M})$ reduziu em aproximadamente $60 \%$ a amplitude da contração atrial quando comparado com o controle. Tal efeito foi parcialmente revertido (aproximadamente $75 \%$ ) após a remoção da droga, quando comparado com o controle. Na Figura 1D é possível observar a curva de concentração-efeito do AE sobre a contratilidade $(n=4)$, o qual apresentou uma $I C_{50}$ (concentração que induz metade do efeito máximo) de $558 \pm 24,06 \mu \mathrm{M}$ e efeito máximo $=100 \%$.

\section{Efeito do AE Sobre a $\mathrm{I}_{\mathrm{Ca}, \mathrm{L}}$ em Cardiomiócitos Isolados}

A Figura 2A mostra o traçado representativo da $\mathrm{I}_{\mathrm{Ca}, \mathrm{L}}$ medida experimentalmente. Na figura 2B pode ser observado o curso temporal do efeito do AE sobre a amplitude da $\mathrm{I}_{\mathrm{Ca}, \mathrm{L}^{\circ}}$. Na Figura 2C é possível visualizar traçados representativos da $\mathrm{I}_{\mathrm{Ca}, \mathrm{L}}$ na situação controle (ausência da droga) e na presença de 10, 700 e $3.000 \mu \mathrm{M}$ de AE. Na Figura 2D percebe-se que a exposição ao $\mathrm{AE}$ reduziu a amplitude da $\mathrm{I}_{\mathrm{Ca}, \mathrm{L}}$ de modo dependente de concentração $\left(\mathrm{IC}_{50=} 1.337 \pm 221 \mu \mathrm{M}\right)$.

\section{Discussão}

Os resultados aqui apresentados demonstram que o $\mathrm{AE}$ reduz a força de contração do músculo atrial de cobaia de modo dependente de concentração. Também foi observado que o AE inibe os canais de $\mathrm{Ca}^{2+}$ do tipo $\mathrm{L}\left(\mathrm{Ca}_{\mathrm{v}} 1.2\right)$ em cardiomiócitos.

O AE apresentou um efeito cardiodepressor sobre o inotropismo atrial. Apesar de não existir dados na literatura que demonstrem o efeito cardiodepressor do $A E$, dados de seus análogos, como o eugenol, estão disponíveis. O eugenol,

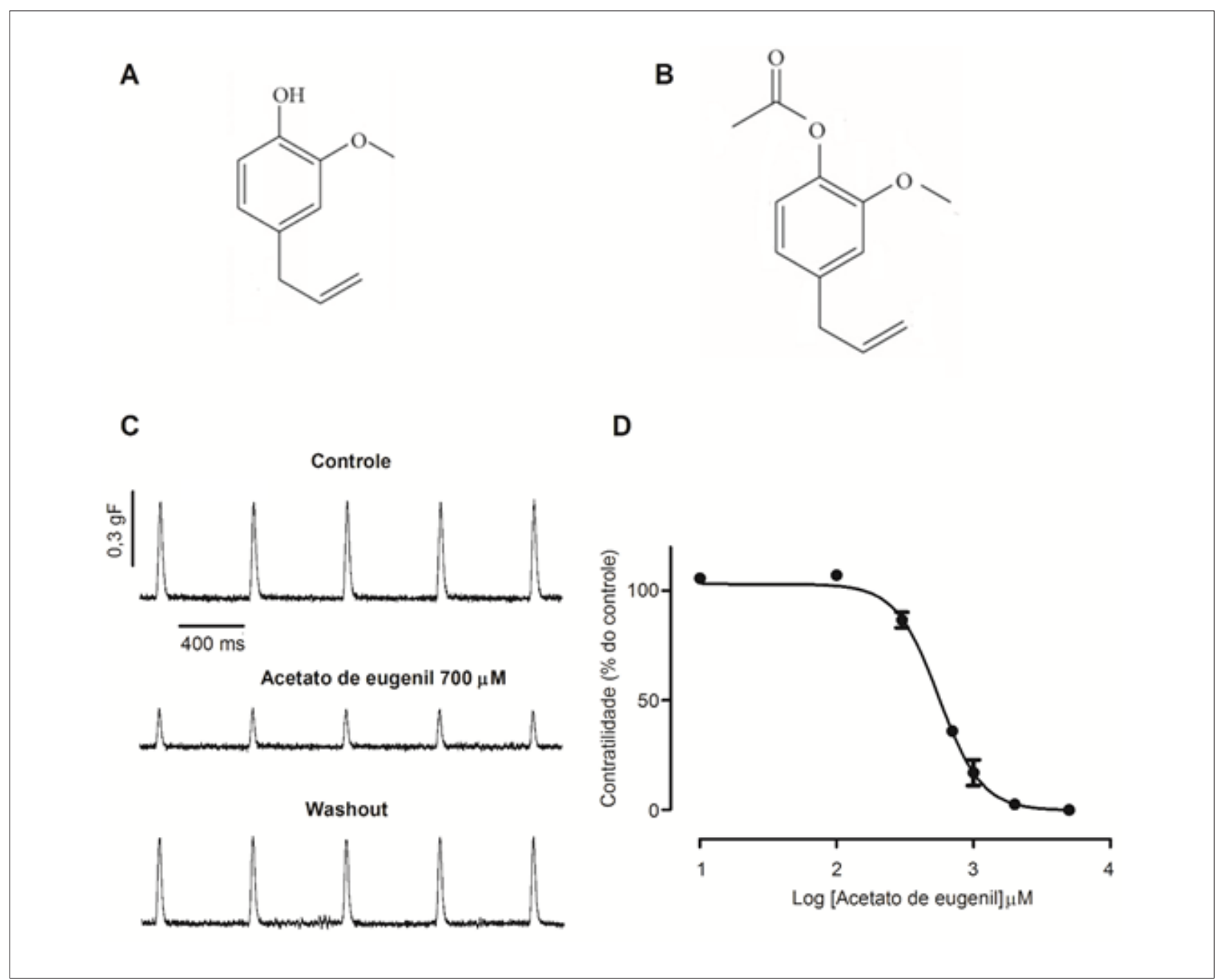

Figura 1 - Efeito do acetato de eugenil (AE) sobre a contratilidade do miocárdio atrial. A) Estrutura do eugenol. B) Estrutura do AE. C) Traçados representativos da contratilidade atrial na situação controle, na presença de $700 \mu M$ de $A E$, e após 10 minutos do washout. D) Curva concentração-efeito inotrópico negativo do $A E$ ( $n=4$ ). 


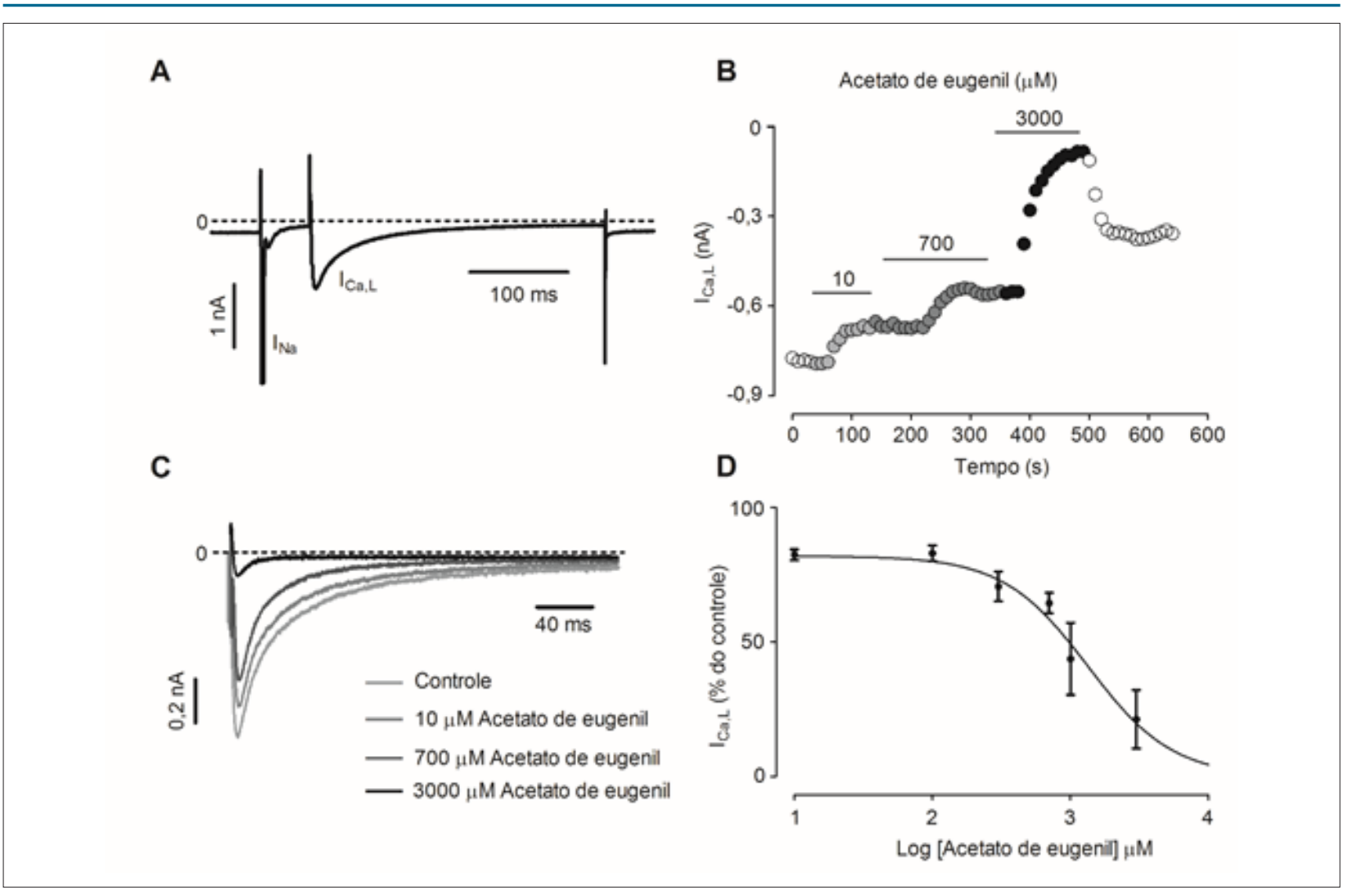

Figura 2 - Efeito do AE sobre a $I_{C a, L}$. A) Corrente iônica obtida experimentalmente. B) Curso temporal do efeito do AE sobre a $I_{\text {Ca, }}$. C) Traçados representativos da $I_{\text {Ca, }}$ no controle e na presença de diferentes concentrações de $A E$. Alinha tracejada indica zero de corrente $D$ ) Curva concentração-efeito do $A E$ sobre a $I_{C a, L}$ em cardiomiócitos ( $n=4$ ).

de modo semelhante à droga aqui investigada reduz a força de contração do miocárdio ventricular de cobaia ${ }^{5}$ e rato, ${ }^{4}$ corroborando os dados aqui achados.

A força da contração do miocárdio correlaciona-se com alterações da amplitude do transiente de $\mathrm{Ca}^{2+}$ que é determinada pelo influxo de $\mathrm{Ca}^{2+}$ através dos canais de $\mathrm{Ca}^{2+}$ presentes no sarcolema, bem como pela quantidade de $\mathrm{Ca}^{2+}$ liberada pelo retículo sarcoplasmático (RS) no processo denominado de acoplamento excitação-contração. A despolarização sarcolemal leva a abertura dos canais de $\mathrm{Ca}^{2+}$ do tipo $\mathrm{L}$ durante a fase do platô do potencial de ação, o que leva a uma corrente de entrada de $\mathrm{Ca}^{2+}$. Esse influxo de $\mathrm{Ca}^{2+}$ estimula a liberação do $\mathrm{Ca}^{2+}$ armazenado no $\mathrm{RS}$, processo conhecido como liberação de $\mathrm{Ca}^{2+}$ induzida por $\mathrm{Ca}^{2+}$, que induz na contratilidade cardíaca. ${ }^{7}$ Dessa forma, mecanismos que alteram o manejo intracelular de $\mathrm{Ca}^{2+}$ estão envolvidos na regulação da contratilidade no músculo cardíaco.

Para tentar explicar o inotropismo negativo do AE sobre o músculo cardíaco foi verificado sua ação sobre a amplitude da $\mathrm{I}_{\mathrm{C}, \mathrm{L}}$ em cardiomiócitos isolados Os achados indicam que o AE reduz a amplitude da $\mathrm{I}_{\mathrm{Ca}, \mathrm{L}}$, efeito que pode estar associado à ativação de receptores que modulam a $\mathrm{I}_{\mathrm{Ca}, \mathrm{L}}$ e/ou ao bloqueio direto desses canais. Este mecanismo pode ser o responsável pela redução da força induzida pelo $\mathrm{AE}$, uma vez que leva a redução da liberação de $\mathrm{Ca}^{2+}$ pelo $\mathrm{RS}$.
Sensch et al., ${ }^{4}$ ao estudar as propriedades farmacológicas do eugenol, quimicamente semelhante ao $\mathrm{AE}$, demonstraram que essa substância deprime a força de contração atrial por reduzir o influxo de $\mathrm{Ca}^{2+}$ nos cardiomiócitos. Nesses experimentos, foi observado que o eugenol apresenta uma $\mathrm{IC}_{50}$ de $127 \mu \mathrm{M}$, valor menor do que a IC $\mathrm{C}_{50}$ do $\mathrm{AE}(1.337 \mu \mathrm{M})$. Esses dados sugerem que o eugenol é mais potente em bloquear a corrente de $\mathrm{Ca}^{2+}$ do que o AE.

\section{Conclusão}

O AE apresenta efeito cardiodepressor que pode ser explicado, pelo menos em parte, pela inibição do $\mathrm{Ca}_{\mathrm{v}} 1.2$.

\section{Agradecimentos}

FAPESP 2014/09861-1 and PICIN/UNEB.

\section{Contribuição dos Autores}

Concepção e desenho da pesquisa e Análise estatística: Marques LP, Gondim ANS; Obtenção de dados: Marques LP, Beserra SS, Gondim ANS; Análise e interpretação dos dados: Marques LP, Roman-Campos D, Gondim ANS; Obtenção de financiamento e Revisão crítica do manuscrito quanto ao conteúdo intelectual importante: Roman-Campos D; Redação do manuscrito: Roman-Campos D, Gondim ANS. 


\section{Potencial Conflito de Interesses}

Declaro não haver conflito de interesses pertinentes.

\section{Fontes de Financiamento}

O presente estudo foi financiado pelo Programa Interno de Iniciação Científica da UNEB (PICIN/UNEB) e Fundação de Amparo de Pesquisa de São Paulo (Fapesp).

\section{Referências}

1. Wang H, Naghavi M, Allen C, Barber RM, Bhutta ZA, Carter A, et al. Global, regional, and national life expectancy, all-cause mortality, and cause-specific mortality for 249 causes of death, 1980-2015: a systematic analysis for the Global Burden of Disease Study 201. The lancet. 2016; 388(10053):1459-544.

2. Khalil AA, urRahman U, Khan MR, Sahar A, Mehmoodac T, Khan M. Essential oil eugenol: sources, extraction techniques and nutraceutical perspectives. RSC Advances. 2017;7(52):32669-81.

3. Damiani CEN, Moreira CM, Zhang HT, Creazzo TL, Vassall DV. Effects of eugenol, an essential oil, on the mechanical and electrical activities of cardiac muscle. J Cardiovasc Pharmacol. 2004;44(6):688-95

4. Sensch O, Vierling W, Brandt W, Reiter M. Effects of inhibition of calcium di and potassium currents in guinea-pig cardiac contraction: comparison

\section{Vinculação Acadêmica}

Não há vinculação deste estudo a programas de pósgraduação. of $\beta$-caryophyllene oxide, eugenol, and nifedipine. Br J Pharmacol. 2000; 131(6):1089-96.

5. Choudhary R, Mishra KP, Subramanyam C. Interrelations between oxidative stress and calcineurin in the attenuation of cardiac apoptosis by eugenol. Mol Cell Biochem. 2006;283(1-2):115-22.

6. Shioya T. A simple technique for isolating healthy heart cells from mouse models. J Physiol Sci. 2007;57(6):327-35.

7. Eisner DA, Caldwell JL, Kistamás K, Trafford AW. Calcium and excitationcontraction coupling in the heart. Circ Res. 2017;121(2):181-95.

8. amill OP, Marty A, Neher E, Sakmann B, Sigworth FJ. Improved patchclamp techniques for high-resolution current recording from cells and cell free membrane patches. Pflugers Arch. 1981;391(2):85-100. 